\title{
LA DINÁMICA METROPOLITANA EN ESPAÑA. ANÁLISIS ESTADÍSTICO Y CARTOGRÁFICO DE LOS MUNICIPIOS A PARTIR DE LA POBLACIÓN Y LA VIVIENDA*
}

\author{
O. de Cos Guerra \\ Dpto. de Geografía Urbanismo y Ordenación del Territorio. \\ Universidad de Cantabria \\ olga.decos@unican.es \\ Escuela de Caminos, Canales y Puertos. Universidad de Cantabria \\ 39005-Santander
}

\begin{abstract}
Resumen: El estudio de la dinámica demográfica y de viviendas a escala municipal en España pone de relieve la existencia de aureolas supra-municipales organizadas en torno a los principales municipios urbanos, en los cuales predomina la inmigración intra-provincial y la proporción de viviendas de obra nueva. Asimismo, la selección de variables sociodemográficas significativas permite resaltar comportamientos relativamente homogéneos en las áreas de influencia de los principales sistemas urbanos. Estos aspectos son los que desde una perspectiva geográfica se incorporan en el presente estudio ${ }^{1}$, sobre la base de la importancia del papel ejercido por las nuevas promociones de viviendas en los procesos de redistribución interna.
\end{abstract}

Palabras clave: Redistribución, dinámica demográfica, área metropolitana, SIG.

Abstract: The analysis of demographic dynamic on municipal scale in Spain shows the existence of organized aureoles around the main urban municipalities.

Likewise, the selection of significant sociodemographic variables lets to stand out relatively homogeneous behaviors in the areas of influence of the main urban systems. These aspects are the ones that since a statistical and cartographic perspective are incorporated in the present stucly. It is based on the base of the importance of the new promotions in the internal processes of redistribution.

Keywords: Redistribution, demographic dynamic, metropolitan area, GIS.

\footnotetext{
* Recibido: 25-09-06. Aceptado: 5-06-07.

${ }^{1}$ El presente artículo tiene su origen en la investigación desarrollada para el estudio clel contexto espanol en la tesis doctoral "La organización del espacio social del área metropolitana de Santánder", dirigicla por el profesor Dr. Pedro Reques Velasco y leída en julio de 2005 en el Departamento de Geografía, Urbanismo y Ordenación del Territorio de la Universiclad de Cantabria.
} 


\section{Introducción}

El análisis de la ocupación del territorio, la configuración de ciudades, sus áreas de influencia y sistemas metropolitanos, constituye un objeto de estudio destacado y ampliamente desarrollado, especialmente desde la segunda mitad del siglo XX. Así lo pone de manifiesto H. Capel (2003) en su obra "Una mirada bistórica sobre los estudios de redes de ciudades y sistemas urbanos" donde el autor explica detalladamente la transformación de los trabajos sobre estos ámbitos, desde los primeros centrados en las jerarquías y redes de ciudades a los estudios más recientes.

Las áreas metropolitanas han sido un importante objeto de estudio en sí mismo y lo siguen siendo por la repercusión que estos espacios tienen en el desarrollo económico, social y medioambiental, como queda de manifiesto en el Dictamen del Comité Económico y Social Europeo (CESE, 2004) sobre las áreas metropolitanas europeas, consideradas territorios principales para la formación, investigación, innovación, nudos de transporte $y$ telecomunicaciones... cuestiones por las que en el citado informe señala el interés de definir las áreas metropolitanas en los países de la Unión Europea y analizarlas con un seguimiento periódico.

En España a pesar de no existir consenso ni denominación unívoca de los espacios configurados en torno a los principales núcleos urbanos -aglomeraciones urbanas (Serrano, 2006), grandes áreas urbanas (Ministerio de Vivienda), aglomeraciones metropolitanas (Serra, 2002), áreas metropolitanas, regiones metropolitanas, etc-- destacan numerosos estudios que abordan directamente el fenómeno urbano-metropolitano como objeto de estudio específico e indirectamente -a partir de estudios de caso- en los que los rasgos, criterios e indicadores propios de sistemas concretos son generalizados y planteados como modelos evolutivos.

Parece claro que los rasgos sociodemográficos de los sistemas metropolitanos en España, no deben ajustarse a los límites impuestos por las unidades administrativas que los configuran ya que responden fundamentalmente a una realidad espacial difusa (Ballet y Llop, 2004) que enlaza con el comportamiento clásico planteado por Gale y Atkitson (1979) enunciado como fuzzy logic o lógica difusa, lo que ha dado origen también a líneas de investigación que recurren al concepto urban sprawl (Dominique, 2004) para referirse a las formas de crecimiento urbano.

Por ello, las metodologías de delimitación de áreas metropolitanas han evolucionado desde la clásica lógica booleana -que posibilita modelos cartográficos elementales basados en criterios binarios de perfil metropolitano- a métodos más avanzados como el multicriterio (De Cos, 2005) o metodologías dinámicas (Roca, 2005; Boix, 2006). 


\section{Justificación de objetivos}

El presente estudio tiene por objeto desarrollar un análisis de base municipal sobre la representación espacial de los rasgos sociodemográficos de los espacios metropolitanos en España. En este sentido constituye una lectura basada en una estrategia inversa en la que primero se marcan los municipios objeto de estudio (sobre la base de la contigüidad con los que alojan la ciudad central) poniendo de relieve la relación existente entre la organización de las unidades "diana" predefinidas si se tiene en cuenta la ubicación de los principales sistemas urbanos españoles, junto a condiciones de proximidad de los términos municipales que en torno a ellos se organizan. Posteriormente se analiza si los principales rasgos metropolitanos, analizados desde el punto de vista sociodemográfico y evolutivo, responden a patrones espaciales homogéneos y generalizables, que son sumidos de forma progresiva por áreas de tamaño medio. Con ello se analizan los claros modelos espaciales organizados en torno a las principales aglomeraciones urbanas ${ }^{2}$; pero también se pone de relieve -cartográfica y estadísticamente- los rasgos generados en las áreas urbanas intermedias, de influencia intra-regional, por el dinamismo y repercusión que éstas representan a escala local (Ballet y Llop, 2004).

Conceptualmente es importante precisar que las áreas metropolitanas difícilmente pueden ceñirse a los límites administrativos de los municipios que las integran y que su organización espacial responde más a un planteamiento difuso que categórico, si bien el estudio se desarrolla a partir de estas unidades administrativas por dos cuestiones fundamentales:

- La escala, pues la sección censal -unidad mínima para la que se dispone de información estadística oficial- no sería apropiada para un estudio de conjunto como el que aquí se presenta.

- El carácter normativo, ya que la experiencia de áreas metropolitanas como entidad administrativa local implica la coordinación de los municipios incorporados, que actúan a modo de integrantes de comarca, con lo que -ajustado o no a la realidad territorial de base-, la coordinación interna metropolitana responde en definitiva a la colaboración intermunicipal.

El estudio presentado se basa en el análisis cartográfico y estadístico municipal de rasgos demográficos, de viviendas y hogares en España fundamentalmente a partir de

\footnotetext{
${ }^{2}$ Según el informe titulado "Políticas Urbanas en España" disponible en European Urban Knowledge Network (http://www.eukn.org/espana/spainurbanopolicy/index.html) España cuenta con cuatro grandes áreas urbanas (Madrid, Barcelona, Valencia y Sevilla) a las que se unen nueve áreas urbanas más, de menor entidad, entre 500.000 y 1.000 .000 de habitantes (Bilbao, Málaga, Ovieclo-Gijón-Avilés, Zaragozil, Alicante, Cádiz, Vigo-Pontevedra, Murcia y Las Palmas de Gran Canaria). A éstas se une un conjunto de 35 áreas urbanas con tamaños comprendidos entre 100.000 y 500.000 habitantes y por debajo de este rango con más de 50.000 habitantes aparecen unas 30 áreas más.
} 
los datos del Censo de Población y Viviendas de 2001, generado por el Instituto Nacional de Estadística (INE), de la Estadística de Variaciones Residenciales de 2005 y de las actualizaciones del Padrón de Habitantes.

Ello permite una visión de síntesis que puede resultar representativa, directa o indirectamente, del fenómeno metropolitano en España y su dinámica reciente; para lo que es necesaria la elaboración y selección de indicadores de base municipal que, incorporados a la base de datos y relacionados con la base cartográfica correspondiente, permitan analizar espacialmente la incorporación a la dinámica urbana y metropolitana de los distintos municipios españoles en las últimas décadas, quedando fuera de los objetivos del presente estudio -por cuestiones operativas- otros aspectos no menos importantes, como los culturales, comportamentales, perceptuales, o los estructurales y relativos a la concentración de actividades económicas en ámbitos urbanos.

\section{Los sistemas urbanos en España: antecedentes y tendencia reciente}

España ha experimentado en las últimas décadas un importante desarrollo urbano que se manifiesta en la existencia de una realidad metropolitana supramunicipal, que se va gestando y ampliando espacialmente de forma difusa en las proximidades de los núcleos urbanos, por variadas que sean sus características y por distintos que parezcan los ámbitos en los que se desarrollan. Esta forma de crecimiento origina realidades de difícil sistematización y definición, que han sido objeto de estudio especialmente desde el último cuarto del siglo XX (Capel, 1975; Vinuesa, 1975) hasta la actualidad (Serra, 2002; Feria, 2004; Roca, 2003; Roca et al., 2004; Serrano, 2006; Boix, 2007). Esto ha propiciado que se establezcan -tanto en estudios específicos sobre el hecho metropolitano como a partir de estudios de caso - criterios cualitativos y cuantitativos para definir lo que puede entenderse como ciudad y su ámbito de influencia, así como la diferenciación de los conceptos y denominaciones posibles ${ }^{3}$.

Uno de los criterios más extendidos para la delimitación de lo urbano y su ámbito de influencia es el volumen demográfico, y derivado de él la consideración (en términos relativos) de la densidad de población; si bien para establecer umbrales con-

\footnotetext{
${ }^{3} \mathrm{~J}$. Vinuesa en su artículo titulado "Sobre el concepto de ârea metropolitana" (1975) explicaba la diferenciación de los términos cle área metropolitana, conurbación y aglomeración planteando que "el conceplo de continuidad está referido lógicamente al carácter metropolitano y exige la presencia ininterrumpida de este carácter por todo el área (...) debe entenderse de una forma funcional; la idea de continuidad no se constriñe a las superficies edificadas, sino que valora los demás factores del carácter metropolitano (modo de vida, diversificación, interdependencia, etc.)".
} 
cretos aparecen múltiples propuestas. Además se suele incluir la condición de contigüidad, en la que frecuentemente se delimitan como área metropolitana los municipios colindantes a partir del que contiene la ciudad central, conformando unidades de diversa denominación (área metropolitana, conurbano, aglomeración urbana ${ }^{4}$, etc.). Si bien, a pesar de la variedad de criterios definitorios de lo urbano, no se ha llegado a una definición consensuada y aplicable de forma general para todas las épocas y todos los ámbitos.

Con ello, a pesar de ser un tema ampliamente desarrollado, conceptualmente no está cerrado; así, los enfoques y criterios de delimitación de las áreas metropolitanas son en España variados (Serrano, 2006, 2). En este sentido, la alternativa más frecuente, es la delimitación a partir del municipio que alberga la ciudad central, y de los limítrofes, que cumplan ciertas condiciones, tal como señaló A. Precedo (1986, 125), que utilizó para las áreas metropolitanas no definidas oficialmente, el agregado del municipio central y los periféricos con densidades superiores a $100 \mathrm{hab} . / \mathrm{km}^{2}$, con crecimiento interanual superior al medio de la zona central y ubicados en continuidad.

Destaca, asimismo, la identificación de rasgos definitorios de la estructura urbana desarrollada por J. M. Santos Preciado (2000) que señala la existencia de un elevado volumen de población, con especialización de la función residencial en los núcleos periféricos -lo que origina una importante intensidad de movimientos pendulares diarios entre los lugares de residencia, de trabajo y gestión-, la aparición de nuevos centros de abastecimiento y ocio en emplazamientos periféricos, etc. En definitiva, una serie de rasgos que influyen en la organización de una realidad y una dinámica de carácter supra-municipal en las proximidades de los principales municipios urbanos.

En este contexto, es importante retomar la consideración de las áreas metropolitanas como un tipo específico de región (Capel, 1975, 288); como un espacio en el que ciertos indicadores asumen características semejantes, generando patrones territoriales que responden comportamientos relativamente homogéneos en el conjunto de características estudiadas, especialmente si se hace referencia a las posibilidades de análisis que se derivan del Censo de Población de 2001 (Feria, 2004).

\footnotetext{
${ }^{4} \mathrm{~J}$. Serra en su trabajo "Grandes aglomeraciones metropolitanas europeas. Un ensayo de delimitación" (2002) apuntaba como aglomeración metropolitana: agrupación de municipios urbanos (entendiendo por tales los de densidad superior a 250 babitantes por kilómetro cuadrado), fisicamenie contiguos, siluados en torno a la ciudad central (caracterizada por tener más de 100.000 babilantes y una densidad superior a 1.500 habitantes por kilómetro cuadrado) y que además forma un conjunto urbano con una población superior a 250.000 babitantes y una densidad media superior a los 1.500 babitantes por lailómetro cuadrado...". Cuatro años después, J. M. Serrano refuerza de nuevo la condición de continuidad espacial al apuntar en su artículo "Aglomeraciones y áreas urbanas en España, dimensión y tendencias" la definición de áreas y aglomeraciones urbanas como "aquellas äreas contiguas, constiluidas por uno o varios municipios, en la que la suma de su población residente supera en el allimo año de referencia el umbral de los 100.000 babitantes".
} 
De los estudios existentes, tanto centrados en aspectos conceptuales sobre áreas metropolitanas, como en estudios de caso, se deducen una serie de rasgos que se han manifestado en las últimas décadas en los diferentes sistemas urbanos españoles, con intensidad y contexto cronológico variable, entre los que la dinámica cobra especial protagonismo.

Así, atendiendo a la dinámica demográfica, las principales ciudades prácticamente descle los años 80 -e incluso antes- están experimentando un importante proceso de descentralización ${ }^{5}$, ligado en parte al cambio de pautas residenciales -a la especiali-

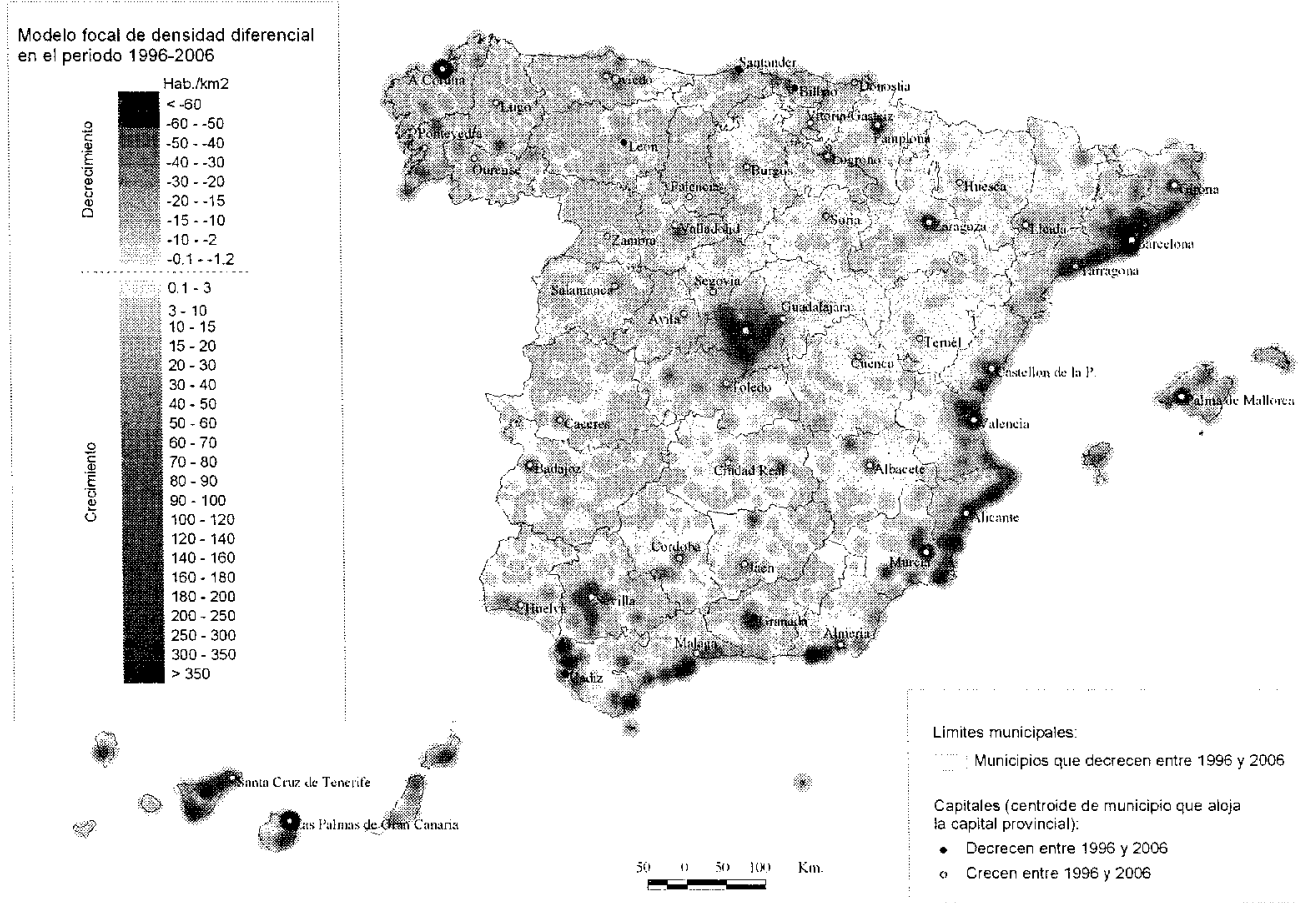

Fuente: INE, Padrones Municipales (1996 y 2006). Elaboración propia.

Figura 1. Modelo de densidad focal (Kernel) de crecimiento de los municipios españoles en el periodo 1996-2006.

\footnotetext{
${ }^{5}$ Es frecuente encontrar en los estudios sobre la dinámica demográfica en las áreas metropolitanas, la utilización de conceptos como desconcentración urbana (Bonvalet, 1989), descentralización (Chesire y Hay, 1985; Diez Orueta, 1991; Reques y Rodríguez, 1996), redistribución poblacional (Fielding, 1989) e, incluso, despoblamiento (Abellán, 1976).
} 
zación residencial periférica- y al declive demográfico de los centros, lo que supone un nuevo ciclo frente a la tendencia centralizadora de décadas anteriores. Este comportamiento iniciado en los sistemas urbanos de mayor tamaño (como Madrid o Barcelona) ha sido asumido progresivamente por sistemas de menor envergadura. El citado proceso descentralizador se ha podido constatar empíricamente en las últimas décadas hasta la referencia del Censo de Población de 2001, si bien a tenor de los últimos datos patronales -y la comparación de éstos con los patronales de 1996 para evitar, en la medida de lo posible, la sobreestimación de la fuente (Godenau, 2004)parece estar despertando un nuevo ciclo en los municipios centrales de los sistemas de mayor tamaño (Figura 1), tal como apunta O. Nel-lo (2004) en su artículo "Cambio de siglo, cambio de ciclo?" al detectar ciertos sintomas de recentralización $(2004,523)$ tras un detallado análisis evolutivo de los principales sistemas metropolitanos españoles. Así, aquellas áreas que décadas atrás fueron pioneras de los procesos de descentralización -de signo contrario a los que acontecían en la ciudad de antes de los 70-, recientemente comienzan a protagonizar un nuevo proceso de crecimiento o, al menos, de reequilibrio demográfico.

El cambio de tendencia apuntado se puede confirmar, si se considera la evolución reciente (2000-2006) de las entidades singulares según los nomenclátores correspondientes (INE). Tal como muestra la Figura 2, la selección de casos realizada permite ver situaciones distintas según el tamaño de la ciudad principal que genera el sistema metropolitano: en Madrid y Barcelona - de primeros sistemas en protagonizar procesos de descentralización- se asiste en los últimos años a un proceso de recuperación de efectivos demográficos o de reequilibrio (con unas tasas de crecimiento medio anual en el periodo del $1,4 \%$ y $1,2 \%$ respectivamente). Esta tendencia progresiva se relaciona con la llegada de población extranjera a los municipios y, en concreto, núcleos y distritos centrales que compensa en cierto modo la salida de residentes de origen nacional, tal como señala E. Pozo $(2005$, 369) para el caso madrileño.

Así, en Madrid, según la Estadística de Variaciones Residenciales elaborada por el INE en 2005 de las casi 116.000 personas nacidas en el extranjero que llegan a la Comunidad de Madrid, el 56\% elige el municipio central. Situación semejante se obtiene en el caso de Barcelona, donde de las 109.000 personas extranjeras que llegan a la provincia, cerca del $50 \%$ residen en el municipio central.

Una situlación bien distinta se muestra en el caso de Santander, ciudad mediana con una evolución reciente descentralizadora -que comenzó con una década de retraso frente a los principales sistemas urbanos- y todavía está inmersa en pleno ciclo de pérdida de efectivos demográficos en la ciudad central (con un ritmo de decrecimiento medio anual entre 2000 y 2006 de $-0,6 \%$ ). Una situación intermedia aparece en el caso del núcleo de Bilbao al manifestarse al final de la serie considerada un repunte, si bien, por la brevedad de la misma no se puede asegurar su mantenimiento en años sucesivos. 
Madrid

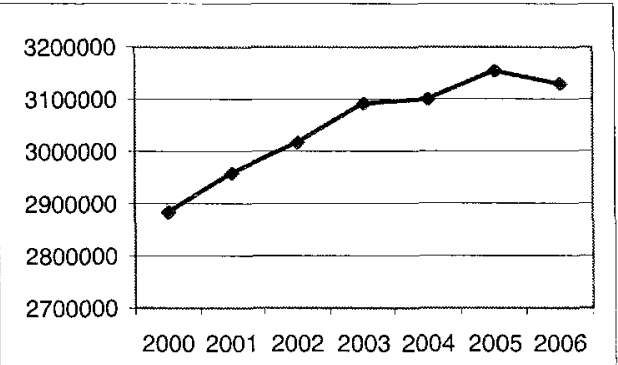

Valencia

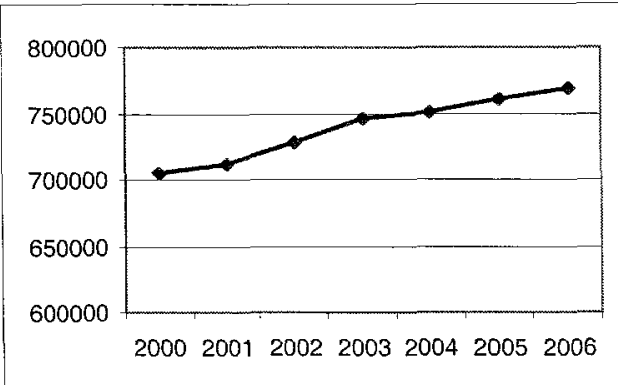

Bilbao

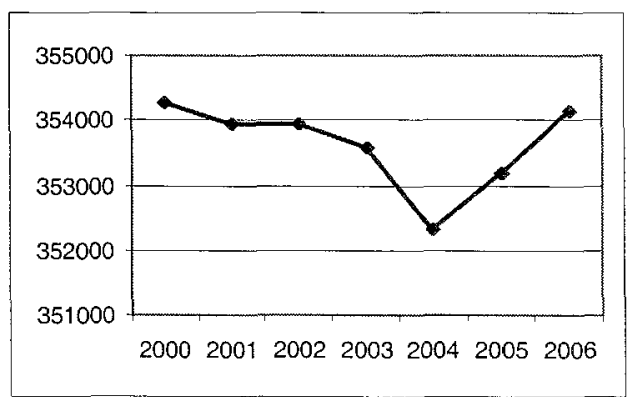

Barcelona

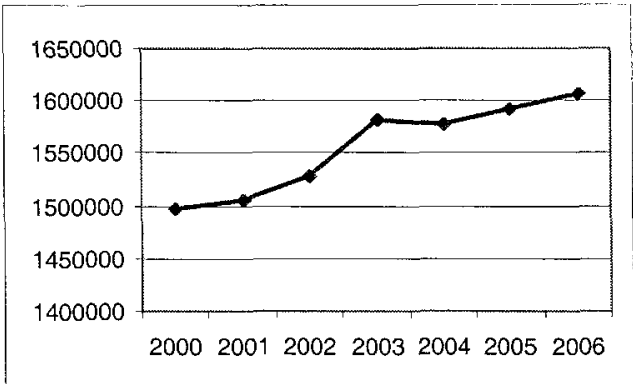

Zaragoza

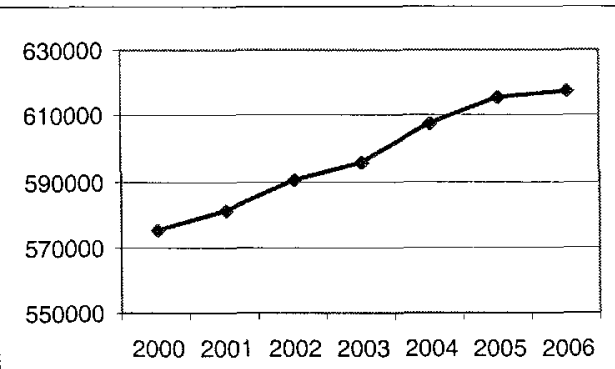

\section{Santander}

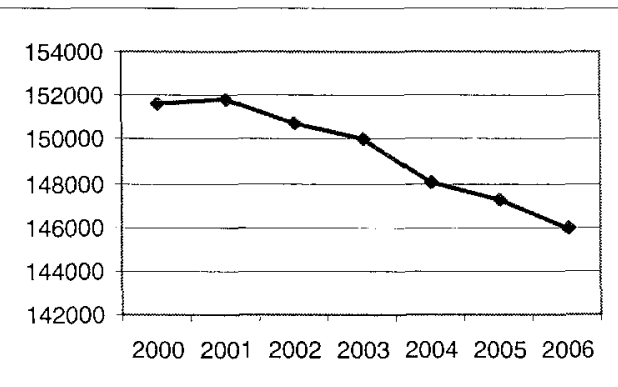

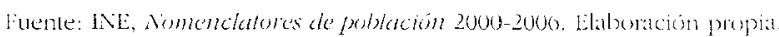

Figura 2. Evolución reciente de la población de las entidades singulares que corresponden con la ciudad central de los sistemas metropolitanos: Madrid, Barcelona, Valencia, Zaragoza, Bilbao, Santander. 
En lo que hace referencia a procesos socio-demográficos, las áreas metropolitanas aparecen como sistemas en los que conviven diversas situaciones, que asociadas al cambio demográfico por éstas experimentado, se asocia de forma sintética a los rasgos que a continuación se exponen: gradación espacial del envejecimiento -envejecimiento de la ciudad central frente al rejuvenecimiento de su área metropolitana-, disminución del tamaño de los hogares e incremento de los individuales y parejas sin hijos, segregación social y procesos de gentrificación o elitización en ciertos sectores centrales (García Herrera, 2001), cambios funcionales y terciarización en las áreas centrales frente a la especialización residencial de la periferia.

\section{Precisiones metodológicas: identificación de los municipios de referencia}

El análisis del perfil de los municipios españoles aquí presentado se desarrolla a partir de un Sistema de Información Geográfica de base municipal que ha permitido, por un lado, la generación de cartografía específica para diferentes periodos y, por otro, la realización de consultas espaciales y cálculos agregados a partir de las variables de base.

Una consulta por atributos fundamental sustenta la identificación inicial de todos los municipios que alojan la capital de cada una de las provincias y los términos municipales de cierta entidad demográfica, criterio discutido, pero frecuentemente utilizado para seleccionar, definir y delimitar ámbitos urbanos; concretamente, se toma como referencia el tamaño mínimo de 50.000 habitantes, umbral ampliamente utilizado desde hace décadas ${ }^{6}$ para la selección de áreas con cierta entidad demográfica, tanto en los estudios sobre áreas urbanas realizados por el Ministerio de la Vivienda, como en trabajos específicos recientes (Boix, 2006, 3).

Fruto de esta consulta se individualiza un total de 115 municipios, que agrupan tan sólo el $6 \%$ de la superficie del país, pero casi el $50 \%$ de la población empadronada en $2006^{7}$.

Posteriormente, a partir de una consulta espacial, se señalan los municipios que limitan en algún tramo con los términos indicados anteriormente, de lo que resulta

\footnotetext{
${ }^{6}$ Ya en 1949 este umbral aparecía asociado al concepto estadístico oficial cle Área Metropolitana Estándar (SMA), que era entendido como el condado o conjunto de condados contiguos con al menos una ciudad de 50.000 habitantes y una densidad superior a unos $60 \mathrm{hab} / \mathrm{km}^{2}$.

${ }^{7}$ Según las cifras del Padrón de Habitantes (INE) el conjunto de municipios incorporados en la primeral consulta espacial presentan un total de 21.643 .985 habitantes.
} 
una selección de 950 municipios aproximadamente, que engloban el 14\% de la superficie del país y prácticamente el $17 \%$ de la población en 2006. De este modo, se definen tres zonas -dos específicas y una tercera por deducción- que quedan organizadas del siguiente modo: los municipios que contienen las propias capitales y los de cierta entidad demográfica; los municipios limítrofes a los anteriores y, finalmente, el resto de municipios ( $80 \%$ de la superficie pero menos del $35 \%$ de la población) que no forman parte de ninguna de las categorías mencionadas.

Sobre los dos sectores definidos inicialmente se centra el presente trabajo, entendiendo por tales conjuntos de municipios aquellos que protagonizan el proceso de metropolización en España por corresponder con municipios centrales (mayores de 50.000 habitantes, tal como se ha señalado con anterioridad), o bien por ubicarse en sus proximidades. Sobre esta base es interesante plantear unidades espaciales que incorporen nuevos aspectos complementarios a los de ubicación y tamaño, de modo que la propia expresión territorial de análisis posteriores permita la identificación de unidades de comportamiento homogéneo, que respondan a los caracteres y procesos metropolitanos, señalados en el apartado anterior.

En esta aproximación inicial, el argumento básico de definición de zonas de interés urbano y metropolitano ha sido el tamaño demográfico y la contigüidad con los municipios centrales. Si bien, en los diferentes estudios realizados en nuestro país en las últimas décadas las aureolas de identificación de áreas urbanas y metropolitanas han sido objeto de diferentes aproximaciones, que a continuación se recogen sucintamente.

Con referencia a la situación española en el contexto urbano europeo el Informe de Reclus realizado en 1989 ya analizaba las ciudades españolas que configurasen aglomeraciones superiores a 200.000 habitantes (Juaristi, 1995). En el citado estudio se elaboró una clasificación basada en ocho niveles de aglomeraciones urbanas, atendiendo a indicadores muy diversos, relativos a la población y su evolución, la presencia de multinacionales, la situación de las infraestructuras o la cualificación de la población. De todas las categorías definidas, España sólo contaba con casos conprendidos entre los niveles 3 y 8 (de entidad decreciente), de modo que en la categoría 3 aparecen únicamente Madrid y Barcelona y en el otro extremo -categoría 8se enmarca la mayoría de ciudades pasando por situaciones intermedias como la de Sevilla y Valencia (ambas en el nivel 5), Bilbao en el 6 y Zaragoza, Málaga y Valladolid, entre otras, en el nivel 7. Así, tal como muestra la Figura 3, sobre la base de las unidades definidas anteriormente (que son objeto de estudio en el presente trabajo) se observa que son limitadas las ciudades consideradas de cierta entidad.

\footnotetext{
${ }^{8}$ RECLUS (1989): Les villes européennes, París: DATAR. Citado en JUARISTI, J. (1995): "El sistema urbano español y sus relaciones con el sistema urbano europeo". En: Las ciudades españolas a finales del siglo XX: I Coloquio de Geografia Urbana. Cuenca: Universidad de Castilla-La Mancha - Madricl: AGE, Pp. 17-27.
} 


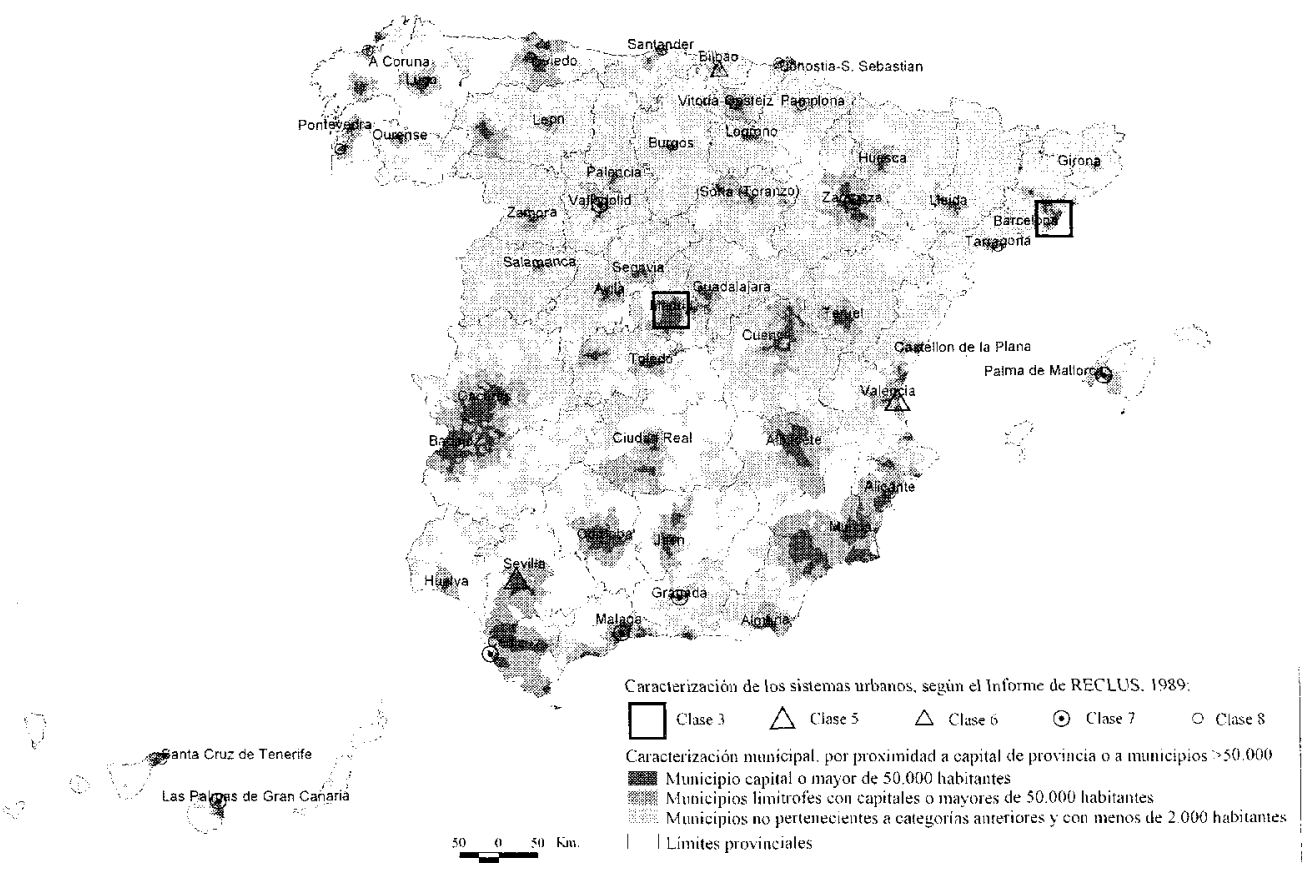

Figura 3. Distribución en España de las tipologias europeas de Reclus para aglomeraciones mayores de 200.000 habitantes y condición de contiguidad con los términos municipales de mayor tamaño.

Fuente: Base en De Cos, O. (2004) y representación cartográfica de símbolos realizada a partir de la clasificación de Reclus citada en Juaristi Linacero, J. (1995): "El sistema urbano español y sus relaciones con el sistema urbano europeo". En: Las ciudades españolas a finales del siglo XX. I Coloquio de Geografia Urbana. Cuenca: Universidad de Castilla-La ManchaMadrid: Asociación de Geógrafos Españoles, Grupo de Geografía Urbana, p. 25.

En 1991, E. González Urruela en su estudio "Industrialización y desarrollo metropolitano en España" basa en la identificación de los principales sistemas urbanos en función de su población, argumentando la industrialización como causa explicativa de la diversidad de situaciones. Sobre esta base la autora identificó cinco grandes áreas metropolitanas -Madrid, Barcelona, Valencia, Sevilla y Bilbao- y otras organizadas en torno a ciudades con más de 150.000 habitantes (Zaragoza, Málaga, Santander, San Sebastián, Valladolid, A Coruña, Gijón, Murcia, Córdoba o Granada, entre otras).

Con los datos disponibles del Censo de Población de 2001 (INE), la situación es analizada por J. Roca et al. (2004) en el trabajo "Las áreas metropolitanas españolas. Evolución 1991-2001" que pone de manifiesto la configuración de siete áreas metro- 
politanas principales: las cinco planteadas en el estudio de González Urruela, a las que se unen otras dos, identificadas en el nivel 7 según el Informe de Reclus (1989): Málaga y Zaragoza, esta última con un crecimiento muy importante en la última década (López Ramón, 2005).

Aproximaciones recientes a la identificación de sectores destacados bajo la dinámica de los principales sistemas urbanos amplían el tamaño y número de sistemas metropolitanos. Según el estudio de J.M. Serrano (2006) "Aglomeraciones urbanas en España, dimensión y tendencias", el sistema urbano reposaría sobre 45 aglomeraciones urbanas (mayores de 100.000 habitantes). Destaca, asimismo, el trabajo de R. Boix (2007) Concepto y delimitación de âreas metropolitanas: una aplicación a las áreas metropolitanas en España, que amplía la identificación de áreas metropolitanas a 67 unidades definidas a partir de iteraciones sucesivas. La organización jerárquica según tamaño demográfico vuelve a estar presente como criterio de identificación, que en el citado estudio se traduce en cuatro niveles, desde el orden A (mayores del millón de habitantes, como son Madrid, Barcelona, Valencia y Sevilla) hasta el orden D (menores de 100.000 habitantes), nivel que quedaría por tanto fuera de las aglomeraciones urbanas definidas por $\mathrm{J}$. M. Serrano.

\section{Criterios de individualización de los municipios incorporados en los sistemas urbanos y metropolitanos}

\subsection{La redistribución intra-provincial y la importancia de la vivienda de reciente construcción}

Son importantes y variados en su enfoque los estudios generales y de caso, en los que se hace referencia a la pérdida de efectivos demográficos en la ciudad central en favor de la corona metropolitana, bien por los precios de la vivienda más asequibles del periurbano, bien atraídos por las tipologías unifamiliares de baja densidad (Rodríguez y Reques, 2000).

Sea cual fuere la causa del proceso y las características de este colectivo, su salida se traduce en una mayor presencia de población que ha nacido en municipios distintos a los de residencia, pero en muchos de los casos pertenecientes a la misma provincia (Feria, 2004). Es por ello que el presente estudio se centra en el conjunto de residentes que, según el Censo de Población y Viviendas de 2001, vive en otro municipio distinto al de nacimiento, pero de la misma provincia de lo que se deduce un indicador de aloctonía (Reques y Rodríguez, 1998) en este caso municipal e intraprovincial. 
De este modo, si la media nacional de residentes en cada municipio que han nacido en otro de la misma provincia es del $25 \%$, en las aureolas definidas de municipios limitrofes a las capitales de provincia o a municipios mayores de 50.000 habitantes esta proporción asciende al $40 \%$ (Tabla 1).

Tabla 1. Proporción de viviendas construidas entre 1997 y 2001 y aloctonía municipal intra-provincial, por ámbitos de estudio.

\begin{tabular}{lcc}
\hline Zonas & $\begin{array}{c}\text { \% Viviendas construidas } \\
1997-2001\end{array}$ & $\begin{array}{c}\text { \% Población nacida en otro } \\
\text { municipio (misma provincia) }\end{array}$ \\
\hline Capital ó $>50.000$ & 9,1 & 17,7 \\
Limítrofes & 10,6 & 40,5 \\
Resto & 5,2 & 27,9 \\
Media nacional & 8,0 & 25,0 \\
\hline
\end{tabular}

Fuente: INE, Censo de Población y Viviendas de 2001. Elaboración propia.

Por otro lado, en muchas ciudades las promociones inmobiliarias de nueva construcción están ocupando, incluso saturando, los espacios periurbanos libres, dependiendo muchas veces de las figuras de planeamiento de los municipios limítrofes con la ciudad central (Santos Preciado, 2001). Ello ha desembocado en una tendencia constructiva que estadísticamente puede reflejarse en una importante proporción de viviendas construidas en los últimos años (Vinuesa, 2004), con patrones de localización justificados por la búsqueda de emplazamientos favorables en cuanto a su proximidad a los principales ejes de comunicación y por su cercanía a elementos de atracción residencial, como los que configuran las grandes superficies comerciales del periurbano.

Para abordar cartográficamente este hecho, se parte de los datos ofrecidos por el Censo de Población y Viviendas de 2001 (INE), agrupando las viviendas censadas que se han construido en el último quinquenio (hasta 2001), que tiene un valor medio municipal en España del $8 \%$, pero que puede alcanzar proporciones medias del $11 \%$ en el corredor limítrofe con la capital provincial o con municipios mayores de 50.000 habitantes.

Más representativo puede resultar aún, imbricar estos indicadores en una representación cartográfica única, en la que se generan ocho categorías distintas de municipios, sobre la base de la combinación del origen de la población y la renovación del parque de viviendas, unidos a la dinámica evolutiva municipal. El resultado conbinado genera zonas progresivas y regresivas con las siguientes particularidades según los otros dos indicadores mencionados anteriormente: vivienda y aloctonía municipal intra-provincial de los residentes (Figura 4). 


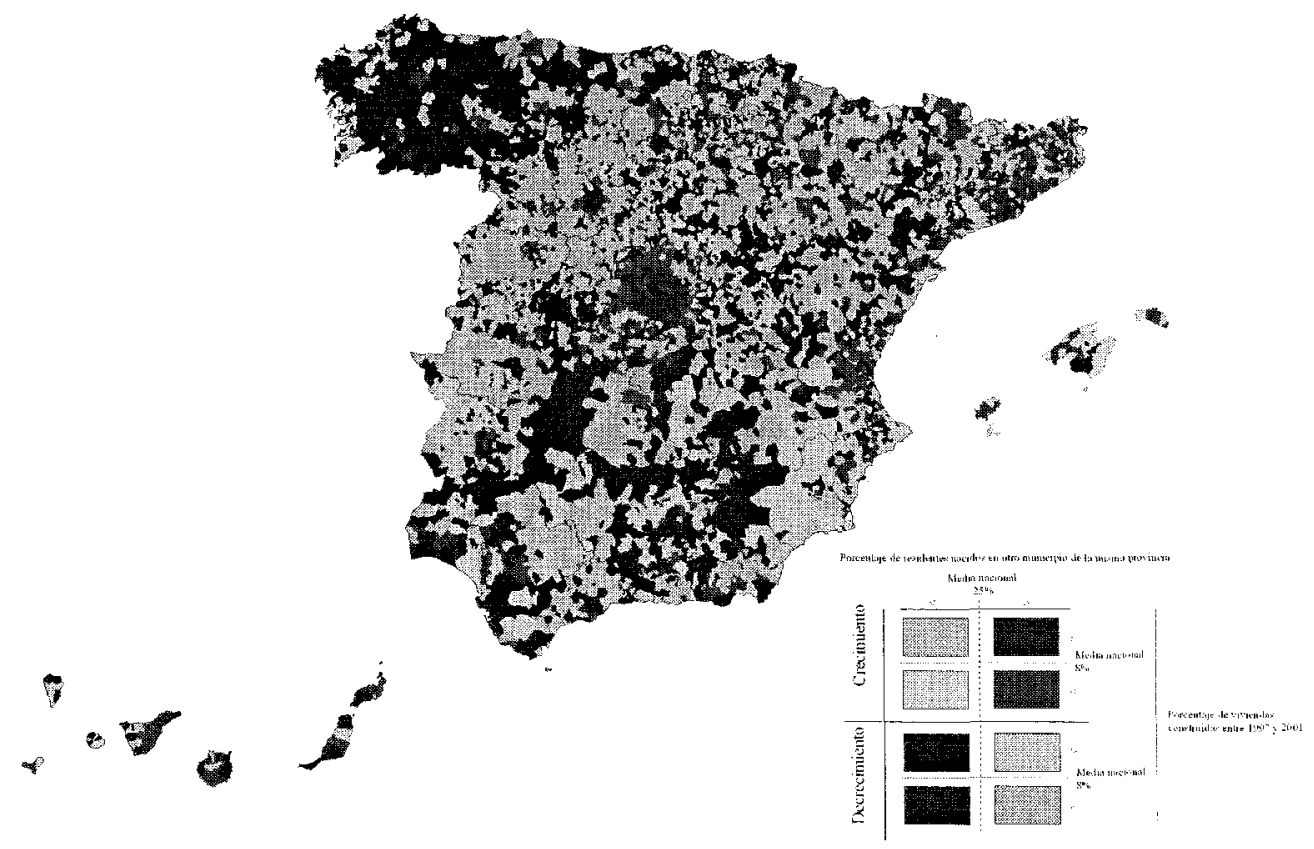

Figura 4. Tipologías municipales según la evolución de la proporción de nuevas viviendas y aloctonía municipal intra-provincial de los residentes.

Fuente: INE, Censo de Población y Viviendas de 2001. Elaboración propia.

Aparecen aproximadamente 900 municipios de perfil metropolitano, caracterizados por una evolución demográfica progresiva en la década 1991-2001, con un porcentaje de viviendas construidas en el último quinquenio superior a la media nacional $(8 \%)$ y un elevado porcentaje de población residente que procede de otros municipios de la misma provincia -que en buena parte de los casos proceden del municipio que aloja la ciudad central-.

Otra categoria destacable corresponde a municipios progresivos desde el punto de vista evolutivo, pero que no cumplen alguna de las otras dos condiciones, bien por tener un parque de viviendas con una dinámica inferior a la media nacional en el último quinquenio (1.071 municipios) o bien porque el origen de población procedente de otros municipios de la provincia no es representativa (243). Ambas categorías se interpretan como parte de las áreas de influencia metropolitana en proceso de incorporación. 
En el polo opuesto a los perfiles mencionados, aparece una categoría de municipios regresivos (más de 1.700), con una proporción de viviendas nuevas y residentes de otros municipios inferior a la media. Estos rasgos coinciden con dos tipologias de municipios relativamente claras, cuya individualización se basa en la localización espacial relativa y en el tamaño demográfico: de un lado, los municipios que alojan la ciudad central (muchos de ellos todavía en fase de descentralización en 2001) y, de otro, los municipios pertenecientes a áreas rurales de bajo volumen de población con procesos de despoblación.

Con comportamiento regresivo, pero con rasgos híbridos de evolución demográfica y dinámica constructiva, aparecen dos tipologías de municipios que en unos casos presentan valores superiores a la media en el porcentaje de viviendas nuevas (258 municipios) y en otros, en el del origen de la población procedente de otros municipios de la misma provincia (más de 2.500). Éstos se encuentran distribuidos en los espacios intermedios entre áreas de influencia de diferentes sistemas urbanos, especialmente en la mitad norte y al sur de Madrid entre Toledo y Ciudad Real.

Aparecen más de 450 municipios que destacan como destino turístico de segunda residencia, con un perfil caracterizado por una evolución estancada o, en algunos casos, regresiva, pero con una proporción de viviendas nuevas elevada (que protagonizará vínculos al municipio por segunda residencia) y con una proporción de población procedente de otros municipios de la misma provincia superior a la media, ligado en parte al atractivo de estos municipios como zonas residenciales para población de la propia provincia.

Si representativos son los resultados cartográficos de conjunto obtenidos, aún lo son más si se estudian los detalles específicos de algunas áreas metropolitanas ya consolidadas -como Madrid o Barcelona-, así como en otras de menor tamaño, y en distintas fases de desarrollo.

En el caso de Madrid o Barcelona aparece el municipio central como un espacio regresivo hasta 2001 aproximadamente (pérdida de efectivos demográficos), con escasa proporción de viviendas nuevas (entendiendo por tales las construidas entre 1997 y 2001) y con una proporción de residentes procedentes de otros municipios de la provincia inferior a la media, lo que deja entrever que no se consolidan como espacios de inmigración intra-provincial, aún habiéndolo sido en periodos anteriores.

Como si de una barrera física se tratase, el comportamiento de la ciudad central se rodea espacialmente de un conjunto de unidades municipales que atraen a los nuevos residentes, convirtiéndose en focos de atracción demográfica y, con ello, en espacios muy progresivos. 
Destaca especialmente, en el caso de Madrid, la organización de dos coronas municipales en torno al espacio central.

La primera corona aparece configurada por todos los municipios directamente influidos por la dinámica metropolitana reciente, que han experimentado un fuerte impulso constructivo, materializado en una importante proporción de viviendas nuevas y que paralelamente aparecen como lugar de residencia de individuos que proceden de otro municipio de la misma provincia, en buena parte protagonizado por traslados del municipio central, como ponen de manifiesto los microdatos de la Estadistica de Variaciones Residenciales generada por el INE: de las bajas experimentadas en el municipio central de Madrid en el año 2005, prácticamente la mitad corresponden a variaciones con nuevo alta en otro municipio de la misma provincia. Si se tiene en cuenta que la fuente ofrece sólo el detalle del municipio de alta si éste cuenta con más de 10.000 habitantes, podremos observar cómo de las más de 45.000 variaciones internas con origen en el municipio central responden a municipios progresivos definidos en la tipología presentada como "municipios de perfil metropolitano" (Figura 5).

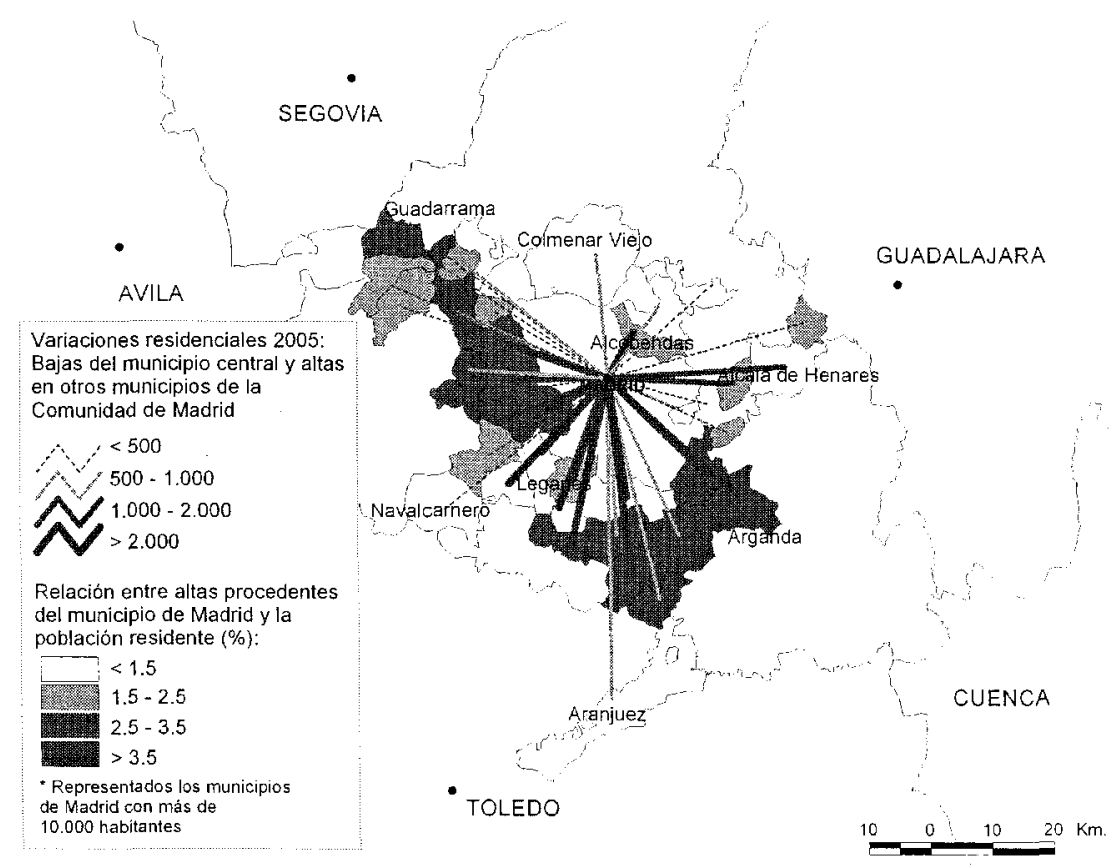

Figura 5. Variaciones residenciales internas en la Comunidad de Madrid: destino de las bajas residenciales del municipio central con destino en otros municipios de la Comunidad. 
Muchos de estos nuevos residentes de los municipios del periurbano trabajan en el municipio central y, por ello, han buscado localizaciones residenciales cercanas, elegidas en un equilibrio dominado por el precio del suelo y la viabilidad (en tiempo) de los desplazamientos pendulares diarios.

A continuación de la aureola descrita se organiza una segunda corona de municipios, menos uniforme, donde hay una presencia incipiente de residentes que proceden de otros municipios de la misma provincia, pero con una proporción de viviendas nuevas inferior a la media, con lo que se puede prever que estos municipios vayan incorporándose en su evolución a la corona mencionada anteriormente, sencillamente cuando la dinámica constructiva de obra nueva alcance mayor protagonismo que el que actualmente tiene.

Con un área de influencia menor, pero con comportamiento y perfil semejante, el modelo se reproduce en ciudades de menor tamaño y de distinta base económica, como es el caso de Bilbao, Santander o Cádiz, todas ellas con un centro regresivo, con escasa proporción de vivienda nueva y poca población procedente de otros municipios, que se rodea de una serie de municipios dinámicos y con un perfil progresivo desde el punto de vista evolutivo y con cierto dinamismo constructivo.

Un comportamiento especial presenta el área de Zaragoza en el cual, el tamaño del municipio central impide analizar los procesos de descentralización (Nel.Lo, 2004, 531), apareciendo como un término progresivo en el periodo 1991-2001, si bien en buena parte de los municipios limítrofes se detectan elevados niveles de aloctonía municipal y una proporción de viviendas de obra nueva por encima de la media, io que es común a los otros casos presentados. Asimismo, el municipio de Zaragoza al margen de su dinámica evolutiva positiva, atendiendo al origen de la población presenta rasgos semejantes a los de otros municipios centrales: una baja proporción de población procedente de otros términos municipales de la provincia.

\subsection{La importancia de los movimientos pendulares diarios de la población ocupada}

Las migraciones interiores en las áreas de influencia de los principales sistemas urbanos y la tendencia a optar por lugares de residencia no coincidentes con el lugar de trabajo, en buena parte de los casos en otro municipio de la provincia (el $28 \%$ de la población ocupada según el Censo de Población de 2001), genera importantes movimientos pendulares diarios, que en las áreas intermedias se producen de manera muy concentrada tanto espacial como temporalmente, en ciertas franjas horarias. Estos desplazamientos por motivos laborales entre municipios de la misma provincia son relativamente cortos en tiempo -el $30 \%$ tarda menos de 20 minutos y el $27 \%$ 
entre 20 minutos y media hora- y se realizan prácticamente en el $75 \%$ de los casos en coche, quedando el uso del autobús con una representación inferior al $8 \%$ y el tren menor al $4 \%$. Según el estudio realizado por J. Gutiérrez Puebla y J.C. García Palomares (2005) se trata de un escenario de aumento de desplazamientos en transporte privado, favorecido por factores muy diversos entre los que los autores destacan cuestiones como el crecimiento disperso y fragmentado de las ciudades, el aumento del nivel de renta o el incremento de la motorización.

Como consecuencia de ello, se genera con frecuencia atascos en las vías principales de entrada y la salida a los principales polos de concentración de actividad laboral y en los municipios que configuran sus cuencas de empleo, que asumen la mayor parte de los desplazamientos realizados por la población ocupada que se dirige del lugar de residencia al de trabajo o viceversa.

En relación a la movilidad, un dato a tener en cuenta es el de la población vinculada, ofrecido por el Censo de Población de 2001, según el cual en España hay un total de 11.620.201 individuos vinculados a otro municipio en el que no residen, de los cuales 5.079 .853 están vinculados por trabajo y 1.190 .915 por estudios, de lo que se deriva un colectivo de casi 6.300 .000 individuos que se desplazan diariamente a otro municipio para trabajar o estudiar.

Retomando la organización de municipios propuesta en este trabajo -municipios centrales (capitales de provincia y mayores de 50.000 habitantes) y limítrofes- es importante destacar que más de la mitad de la población vinculada por trabajo y estudios a otro municipio distinto al de residencia en España, se encuentra ligada a uno de los 115 municipios considerados centrales y casi el $20 \%$ con alguno de los municipios limítrofes en torno a ellos; cuestión que refuerza la concentración de los desplazamientos de movilidad obligada diaria.

\section{Conclusión}

Las áreas metropolitanas están experimentando diferentes procesos y tendencias que derivan en características funcionales, sociodemográficas y espaciales específicas reconocibles, incluso, en un análisis de base municipal.

A partir de los rasgos evolutivos, de construcción de viviendas y del origen de la población se detecta en España una organización supra-municipal que pone de relieve la configuración de rasgos metropolitanos relativamente homogéneos en los municipios próximos a los principales núcleos urbanos, que configuran su área de influencia inmediata. Para los municipios limítrofes con los que alojan la ciudad cen- 
tral se comprueban diferentes niveles de consolidación de estos los rasgos básicos metropolitanos (dinamismo constructivo y atracción de población que está vinculada con la ciudad central o con otros núcleos importantes del sistema), algo que se encuentra en la mayoría de los casos muy relacionado con la entidad demográfica de la ciudad que genera y dinamiza el área metropolitana, así como con la etapa en la que ha comenzado su proceso de metropolización.

Destaca especialmente el caso de Madrid y Barcelona, en cuanto que vienen configurando los ámbitos más avanzados, mientras que otras ciudades de tipo medio o intermedio van experimentando los mismos procesos que los principales sistemas mencionados -junto a Valencia, Bilbao, Sevilla, Málaga o Zaragoza-, si bien, con menor intensidad y extensión, así como con cierto retraso cronológico, que podría estimarse, según los casos, como término medio en una década.

Las cuestiones señaladas, no hacen sino abundar en la idea de que los ámbitos urbanos van asumiendo progresivamente el mismo patrón evolutivo; si bien los ritmos pueden variar de unos casos a otros. En ello influyen de modo determinante diversos factores de difícil sistematización -urbanísticos, políticos, económicos, culturales...- que, si bien no se han incorporado en el desarrollo empírico de este trabajo, sí pueden resultar determinante para estudios de caso a escalas de detalle.

En suma, a pesar de las dificultades manifiestas para la definición-delimitación de áreas metropolitanas, es posible plantear algunas cuestiones generales, como la interpretación de las mismas como espacios dinámicos, de límites difusos y cambiantes, con una organización funcional supra-municipal, y en las que cobran especial importancia los movimientos internos, sobre todo los movimientos pendulares diarios, pero también externos con la región en la que se enmarca. Son áreas en las que las comunicaciones cobran un protagonismo especial y en las que se está produciendo un rápido cambio, una expansión territorial difusa que comienza a convivir en los últimos años con nuevas tendencias de compensación del decrecimiento de los espacios centrales, pero no del proceso de salida de ciertos sectores de población residente en el municipio central. En definitiva, espacios en continua evolución que es necesario analizar para detectar nuevas pautas que pueden marcar la tendencia y perfil de los diferentes sistemas españoles en los próximos años.

\section{Bibliografía}

Bellet Sanfeliu, C.; Llop Tomé, J. M. y (2004):

"Miradas a otros espacios urbanos: las ciudades intermedias". Scripta Nova: Revista electrónica de geografia y ciencias sociales, $\mathrm{n}^{\circ} 8, \mathrm{p} .165$.

Boix Doménech, R. (2006): "Las áreas metro- 
politanas en España". En: Desarrollo de regiones y Eurorregiones. El desafio del cambio rural. XXIII Reunión de Estudios Regionales. Asociación Galega de Ciencia Rexional. [Consulta: Marzo, 2007]

http://www.ecap.uab.es/urban/references/2006/06011.pdf

Boix Doménech, R. (2007): "Concepto y delimitación de áreas metropolitanas: una aplicación a las áreas metropolitanas en España”. Seminario Las grandes áreas metropolitanas españolas en una perspectiva comparada. Sevilla, Abril de 2007 [En línea].

Calvo Palacios, J. L. (1999): "Articulación del espacio y estrategias territoriales". Economía aragonesa, $\mathrm{n}^{\circ}$ 6-7 (Ejemplar Dedicado a la XXIV Reunión de Estudios Regionales), pp. 60-76.

Capel Sáez, H. (1975): "La definición de lo urbano". Estudios Geográficos, no 138-139, pp. 265-301.

Capel Sáez, H. (2003): "Una mirada histórica sobre los estudios de redes de ciudades y sistemas urbanos". GeoTrópico, no 1, pp. 30-65.

En:

http://www.geotropico.org/1_1_Capel.ht $\mathrm{ml}$ [Consultado: Marzo, 2007].

CESE - Comité Económico y Social Europeo (2004): Dictamen sobre las âreas metropolitanas europeas: repercusiones socioeconómicas para el futuro de Europa (Dictamen de iniciativa). ECO/120. Bruselas, 1 de julio de 2004. [En línea]

Díaz de Rada, V. (2002): Técnicas de análisis multivariante para investigación social y comercial. Ejemplos prácticos utilizando SPSS versión 11. Madrid: Ra-Ma.

De Cos Guerra, O. (2004): "Valoración del método de densidades focales (Kernel) para la identificación de los patrones espaciales de crecimiento de la población en España". Geofocus, no 4, pp. 136-165.

De Cos Guerra, O. (2005): La organización del espacio social del área metropolitana de Santander. Tesis doctoral (inédita). Departamento de Geografía, Urbanismo y Ordenación del Territorio. Universidad de Cantabria.

Dominique, M. y Aguilera, A. (2004): Urban Sprawl, Polycentrism and Commuting: $A$ Comparison of Seven French Urban Areas. Urban Public Economics Review, $n^{\circ} 1$, pp. 93-114.

Escolano Utrilla, S. (2002): "Densidad de población y sustentabilidad en la ciudad de Zaragoza", en Longares Alardeen, L. A. y Peña Monné, J. L. (Ed.): Aportaciones geográficas en memoria del profesor $L$. Miguel Yetano Ruiz. Zaragoza, Departamento de Geografía y Ordenación del Territorio, Universidad de Zaragoza, pp. 173-182.

Federal Register (2000): "Standards for Defining Metropolitan and Micropolitan Statistical Areas". Office of Management and Budget.

Feria Toribio, J. M. (2004): "Problemas de definición de las áreas metropolitanas en España". Boletin de la Asociación de Geógrafos Españoles, $11^{\circ} 38$, pp. 85-99.

Ferrao, J. (2004): "Las regiones metropolitanas como ciudades imaginadas: vivencias, discursos y acción). Ciudad y Territorio. Estudios Territoriales, XXXVI (141-142), pp. 517-522.

Gale, S. y Atkitson, M. (1979). On the set theoretical foundations of the regionalization problem. En: Gale \& Olson (eds.): Pbilosophy in Geography; pp. 65-108. Dordrecht, Reidel.

García Coll, A. (2000): "Las migraciones interiores en España en el siglo XX: del éxodo rural al éxodo urbano y otras nuevas formas de movilidad". A Distancia, vol. 18 , $\mathrm{n}^{\circ}$ 1, pp. 146-154.

García Coll, A. (2005): Migraciones interiores y transformaciones territoriales, Papeles, de economía española, no 104, pp. 76-91. 
García Herrera, L. M. (2001): "Elitización: Propuesta en español para el término gentrificación”. Biblio $3 W, \mathrm{n}^{\circ} 332$.

Godenau, D. y Arteaga Herrera, S. (2004): "Fiabilidad de las cifras censales y patronales en Canarias". En: Nuevo Siglo: nuevos datos, nuevos perfiles. IX Congreso de Población Española. Granada: Universidad de Granada, Departamento de Geografía Humana- Asociación de Geógrafos Españoles, Grupo de Población (Inédito).

González Urúla, E. (1991): "Industrialización y desarrollo metropolitano en España". Ería, no 26 , pp. 199-215.

Gutiérrez Gallego, J. A. y Jaraíz Cabanillas, F. J. (2007): Clasificación de los términos municipales de España a partir de variables demográficas y socioeconómicas, empleando métodos de clasificación basados en redes neuronales artificiales. Mapping, $\mathrm{n}^{\circ} 115$, pp. 26-31.

Gutiérrez Jaimez, R., et al. (2000): Tipificación, caracterización y clasificación de áreas territoriales andaluzas mediante técnicas estadísticas multivariantes. Sevilla: Instituto de Estadística de Andalucía.

Gutiérrez Puebla, J. y García Palomares, J.C. (2005): "Cambios en la movilidad en el área metropolitana de Madrid: el creciente uso del transporte privado". Anales de geografia de la Universidad Complutense, $\mathrm{n}^{\circ} 25$, pp. 331-351.

Juaristi Linacero, J. (1995): "El sistema urbano español y sus relaciones con el sistema urbano europeo". En: Las ciudades españolas a finales del siglo XX: I Coloquio de Geografia Urbana. Cuenca: Universidad de Castilla-La Mancha-Madrid: Asociación de Geógrafos Españoles, Grupo de Geografía Urbana, pp. 17-27.

López Ramón, F. (2005): "Ordenación y organización del territorio metropolitano de
Zaragoza, cuestiones pendientes". Territorio \& Desarrollo Local. II época-monográfico 1, pp. 57-66.

Martínez Medina (2006): "Tipologías municipales según la presencia relativa de inmigrantes". Papeles de geografia, n ${ }^{\circ} 43, \mathrm{pp}$. 61-72.

Méndez Gutiérrez del Valle, R. (2002): "La evolución de los mercados de trabajo metropolitanos: realidades y mitos a partir del ejemplo de Madrid". Scripta Nova. Revista electrónica de geografia y ciencias sociales. IV Coloquio Internacional de Geocritica.

Méndez, R. y Caravaca, I. (1993): Procesos de reestructuración industrial en las aglomeraciones metropolitanas españolas. Madrid: MOPT, Dirección General de Política Territorial y Urbanismo.

Ministerio de Vivienda (2005): Atlas estadistico de las áreas urbanas en España. 2004. Madrid.

Moreno Jiménez, A. (1991): "Modelización cartográfica de densidades mediante estimadores Kernel", Treballs de la Societat Catalana de Geografia, 6, 30, pp. 155170.

Nello, O. (2004): "¿Cambio de siglo, cambio de ciclo? Las grandes ciudades españolas en el umbral del S.XXI". Ciudad $y$ Territorio. Estudios Territoriales, XXXVI (141-142), pp. 523-542.

Pozo Rivera, E. (2005): "Tendencias recientes en la evolución de la población de la Comunidad de Madrid". Anales de geografia de la Universidad Complutense, $\mathrm{n}^{\circ} 25$, pp. 353-379.

Precedo Ledo, A. (1986): "Las modificaciones del sistema urbano español en la transición postindustrial". Estudios Territoriales, $\mathrm{n}^{\circ} 20$, pp. 121-138.

Reques, P. y Rodríguez, V. (1998): Atlas de la población española: análisis de base municipal. Santander: Servicio de Publi- 
caciones de la Universidad de Cantabria y CSIC.

Roca Caldera, J. (2003): "La delimitación de la ciudad: ¿̨una cuestión imposible?". Ciudad y Territorio. Estudios Territoriales, XXXV (135), pp. 17-36.

Roca, J., Burns, M. y Moix, M. (2004): Las áreas metropolitanas españolas. Evolución 1991-2001 [en línea]. Barcelona: Centre de Política de Sòl i Valoracions, Universitat Politécnica de Catalunya. [Consulta: agosto de 2004].

http://www.upc.edu/castellano/noticies/acinvestigacion/2004/EstudioAreasMetropolita nas.pdf

Rodríguez, V. y Reques, P. (2000): "La (re)distribución espacial de la población: los cambios a lo largo del presente siglo". A Distancia, Vol. 18, n ${ }^{\circ}$, pp. 155-161.

Santos Preciado, J. M. (2000): "La formación y desarrollo de las áreas metropolitanas". $A$ Distancia, Vol. $18, \mathrm{n}^{\circ} 1$, pp. 162-170.
Santos Preciado, J. M. (2001): "El proceso de distribución espacial de la población en las periferias metropolitanas espanolas (1960-1996)". Anales de Geografía de la Universidad Complutense, $\mathrm{n}^{\circ} 21$, pp. 139181.

Serra, J. (2002): "Grandes aglomeraciones metropolitanas europeas. Un ensayo de delimitación". Papers, n 37.

Serrano Martínez, J. M. (2006): "Aglomeraciones y áreas urbanas en España, dimensión y tendencias: Breves precisiones". Lurralde, 29. [Consulta: Marzo, 2007]

http://www.ingeba.euskalnet.net/lurralde/lur ranet/lur29/29serra/29serra.htm

Vinuesa Angulo, J. (2004): "Población y demanda de vivienda: una relación a considerar". En: La dinámica geodemográfica protagonista del territorio: Actas del VIII Congreso de la Población Española. Santiago de Compostela: Departamento de Xeografia de la Universidad de Santiago de Compostela, pp. 283-290. 